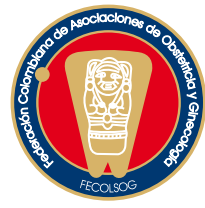

INVESTIGACIÓN ORIGINAL

\title{
ESPECTROSCOPIA DE IMPEDANCIA ELÉCTRICA EN CÁNCER INVASIVO DEL CUELLO UTERINO EN MUJERES DE CALDAS (COLOMBIA), 2008-2009
}

\section{Electrical impedance spectroscopy in cervical cancer in women firom Caldas, Colombia 2008-2009}

Germán Olarte-Echeverri, M.D.*, William Aristizábal-Botero**, Gloria Fátima Osorio-G., M.D.***, Jerónimo Rojas-Díaz****

Recibido: septiembre 30/09 - Aceptado: marzo 8/10

\section{RESUMEN}

Objetivo: obtener las curvas de resistividad eléctrica de tejido cervical en presencia de cáncer invasivo del cuello uterino.

Metodología: estudio de corte transversal en pacientes con carcinoma invasor del cérvix. Se utilizó un medidor de impedancia de tejido (Electrical Impedance Spectroscopy Mk3.5 single channel system), el cual, mediante una sonda tetrapolar con dos electrodos de corriente y dos de voltaje, permite calcular la impedancia de transferencia de los tejidos.

Resultados: se realizaron 102 mediciones y se obtuvieron las curvas de resistividad del tejido cervical en 18 mujeres con edades entre 46 y 72 años $(56+/-9$, media $+/-$ DS). De esta manera, se encontró que los tejidos presentan una curva de resistividad característica para cáncer invasivo de cérvix con parámetros de resistividad promedio $\left(\mathrm{R}_{0}=4,35+/-2,26[\Omega-\mathrm{m}]\right.$, media

* Médico Cirujano, Especialista en Gineco-obstetricia, Universidad de Caldas. Ginecólogo-oncólogo, Pontificia Universidad Javeriana. MSc en Desarrollo Educativo y Social, Universidad Pedagógica Nacional. Programa Cáncer de Cuello Uterino y Cáncer de Mama, Facultad de Ciencias para la Salud, Universidad de Caldas. Manizales (Colombia) Correo electrónico: golarte72@hotmail.com

* Ingeniero Electricista. Candidato a Ph.D en Bioingeniería, Laboratorio de Instrumentación Biofísica, Departamento de Física, Facultad de Ciencias Exactas y Naturales, Universidad de Caldas. Manizales (Colombia).

*** Médico Cirujano, Especialista en Patología Humana. MSc en Educación. Programa Cáncer de Cuello Uterino y Cáncer de Mama, Facultad de Ciencias para la Salud, Universidad de Caldas. Manizales (Colombia).

***** Ingeniero Electrónico, Universidad de Caldas. Manizales (Colombia).
+/- DS y $\mathrm{R}_{\infty}=1,41+/-0,57[\Omega$-m]). Con estos estimativos se obtuvieron los valores de la resistividad de los líquidos extra e intracelular $(\mathrm{R}=4,35+/-2,26$ $[\Omega$-m] y $\mathrm{S}=2,44+/-1,11[\Omega-\mathrm{m}])$.

Conclusión: la espectroscopia de impedancia eléctrica muestra un patrón diferente en tejidos provenientes de carcinoma invasor del cérvix en relación con el tejido cervical sano.

Palabras clave: espectroscopia, análisis espectral, impedancia eléctrica, cáncer de cuello uterino, neoplasias del cuello uterino.

\section{SUMMARY}

Objective: obtaining electrical resistivity curves for cervical tissues in invasive cervical cancer.

Methodology: a tissue impedance meter was used (MK 3.5 Electrical Impedance Spectroscopy, single channel system) in this cross-sectional study of invasive cervical cancer patients; it led to calculating the tissues' transfer impedance by means of a pencil probe incorporating four gold electrodes.

Results: 102 measurements were made and resistivity curves for cervical tissue were obtained in 18 women aged 46 to $72(56 \pm 9$, mean $\pm \mathrm{SD})$. These tissues presented a special resistivity curve for invasive cervical cancer, average resistivity parameters being $\left(\mathrm{R}_{0}=4.35 \pm 2.26[\Omega\right.$-m] and $\left.\mathrm{R}_{\infty}=1.41 \pm 0.57[\Omega-\mathrm{m}]\right)$. Resistivity values for 
intra and extracellular liquids were obtained from those estimations $(\mathrm{R}=4.35 \pm 2.26[\Omega-\mathrm{m}]$ and $\mathrm{S}=2.44 \pm 1.11[\Omega-\mathrm{m}])$.

Conclusion: electrical impedance spectroscopy presented different patterns for invasive cervical cancer tissue and healthy tissue.

Key words: spectrum analysis, electric impedance, uterine cervical neoplasia, cervix neoplasms.

\section{INTRODUCCIÓN}

Latinoamérica es una de las regiones donde la incidencia de cáncer cervical es alta. Dicha patología es el segundo cáncer más común en mujeres y la segunda causa de muerte por cáncer. En el año 2000, se presentaron en la región al menos 76.000 casos de cáncer cervical y 30.000 mujeres fallecieron por esta causa, lo cual representa respectivamente el 13\% y 16\% del total mundial. ${ }^{1}$ En Colombia, la tasa de incidencia en los años 2003 y 2004 fue de 36,8 por $100.000 .^{2}$

La espectroscopia de impedancia eléctrica (EIE) es una técnica que se propone para diferenciar los tejidos escamosos normales de aquellos en estados precancerosos y de cáncer invasivo. Esta técnica se basa en el uso de una sonda tetrapolar del tipo lápiz con electrodos de oro, por medio de la cual es posible obtener el espectro de impedancia eléctrica medida en ohmios-metro $(\Omega$-m) en un rango de frecuencia entre $2 \mathrm{kHz}$ y 1,62 MHz. Para esto, se aplica una corriente de 20 microamperios $(\mu \mathrm{A})$ pico a pico a través de un par de electrodos y se mide sobre el tejido el potencial resultante entre los otros dos electrodos. Posteriormente, se obtiene la razón del potencial medido con respecto a la amplitud de la corriente aplicada y ésta determina la impedancia de transferencia del tejido. Los datos obtenidos en cada uno de los espectros se ajustan por el método de desviación de cuadrados mínimos a una ecuación de Cole-Cole en la que $\alpha=0$, esto hace posible estimar las impedancias a muy bajas y muy altas frecuencias y calcular las resistividades de los líquidos intra (S) y extracelulares (R) en
$(\Omega-\mathrm{m})$, así como la capacitancia de la membrana celular $(\mathrm{Cm})(\mu \mathrm{F} / \mathrm{m}) .^{3-9}$ Las curvas de resistividad de tejidos escamosos cervicales han mostrado que cuando dichos tejidos presentan lesiones que aumentan en grado de severidad (NIC 1 hasta NIC 2-3) su resistividad disminuye y la forma del espectro de impedancia cambia. ${ }^{10}$ Los conceptos teóricos que soportan la aplicación de esta técnica ya han sido previamente publicados. ${ }^{6,10}$

Ahora bien, en la literatura sobre el presente tema no se han presentado las curvas de resistividad eléctrica para tejidos con cáncer invasivo de cérvix y éstas no se han comparado con aquellas que se obtienen de tejidos cervicales sanos. Por lo tanto, este estudio tuvo como objetivo adquirir y caracterizar las curvas de resistividad eléctrica (en ohmios por metro $[\Omega-m]$ ) del tejido cervical en presencia de cáncer invasivo del cuello uterino, en función de la frecuencia de la señal de corriente eléctrica aplicada, la cual fue medida en kilohertz $(\mathrm{kHz})$.

\section{METODOLOGÍA}

\section{Diseño y población}

En este estudio de corte transversal participaron pacientes con cáncer invasivo del cuello uterino, diagnosticado por examen clínico e histopatológico en la sede del Programa de Cáncer de Cuello Uterino y Cáncer de Mama en el Laboratorio de Patología humana de la Universidad de Caldas, sitio de referencia de la red de patología cervical y colposcopia del departamento de Caldas, el cual atiende a pacientes del aseguramiento subsidiado de salud. Por el contrario, se excluyeron a las pacientes con infección cervical activa y con la variedad clínica del cáncer endofítico.

\section{Consideraciones éticas}

Las pacientes fueron seleccionadas mediante un muestreo secuencial por conveniencia y a cada una de ellas se les solicitó un consentimiento informado al comienzo de esta investigación. Finalmente, el estudio fue aprobado por el Comité de Ética Médica de la Facultad de Ciencias para la Salud de la Universidad de Caldas. 


\section{Procedimiento}

A todas las pacientes se les hizo una citología de cuello uterino y la muestra obtenida fue coloreada con la técnica de papanicolaou. Después se les realizó una colposcopia (Leisegang tipo 3153) para determinar los puntos de ubicación de la sonda y la toma de biopsias en las zonas de lesión. Una vez terminada la toma de la biopsia, las zonas fueron cauterizadas con la ayuda de un equipo de radiofrecuencia (Intermed 5000). Además, el cuello uterino de cada paciente fue filmado en video con el fin de correlacionar los resultados histopatológicos y las medidas de impedancia en los puntos de toma de las biopsias. Después de esto, las muestras recopiladas en las biopsias fueron deshidratadas y embebidas en parafina, a cada una se le realizaron cortes de 7 micras y fueron coloreadas por la técnica de hematoxilina y eosina. Finalmente, se analizaron las muestras de las citologías y de las biopsias al microscopio de luz y se obtuvieron fotografías de las placas.

Las medidas de la EIE fueron obtenidas utilizando un medidor de impedancia de tejido (Electrical Impedance Spectroscopy Mk3.5 single channel system) diseñado y construido por el Departamento de Física Médica e Ingeniería Clínica de la Universidad de Sheffield (Inglaterra). Este equipo emplea una sonda tetrapolar de 5,5 $\mathrm{mm}$ de diámetro con cuatro electrodos de oro de $1 \mathrm{~mm}$ de diámetro, espaciados simétricamente sobre un círculo de 1,65 mm de radio. ${ }^{6}$ Dos electrodos aplican una corriente $20 \mu \mathrm{A}$ pico a pico y los otros registran la diferencia de potencial entre dos puntos del tejido afectados por el campo de corriente. El cociente entre el voltaje registrado y la corriente aplicada permite calcular la parte real e imaginaria o la magnitud y fase de la impedancia de transferencia. La sonda eléctrica fue calibrada antes de las mediciones utilizando una solución salina de resistividad conocida (10 $\Omega$-m), medida con un conductímetro comercial (Schott Gerätte Handylab LF12). Los valores de la parte real e imaginaria de la impedancia compleja se obtienen con el equipo por un barrido de 30 frecuencias en un rango entre $2 \mathrm{kHz}$ y 1,62 MHz. El equipo presenta como resultado los espectros de magnitud y fase para la resistividad del tejido. Los valores obtenidos de la parte real e imaginaria de la impedancia, a cada frecuencia, fueron usados para obtener una curva de resistividad promedio con sus correspondientes barras de desviación estándar.

Por otro lado, las curvas de tejido cervical sano con las cuales se compararon las curvas de cáncer invasivo fueron obtenidas en estudios realizados previamente por el grupo de investigación y validadas con la literatura.

\section{Variables a medir}

Se evaluaron la resistividad a baja frecuencia $R_{0}$ y a alta frecuencia $R_{\infty}$, las cuales se obtuvieron mediante un ajuste por regresión de cuadrados mínimos a un modelo de Cole-Cole. A partir de estos parámetros se calcularon la resistividad del líquido extracelular (R), la resistividad del líquido intracelular (S), la capacitancia de la membrana celular $(\mathrm{Cm})$ y la frecuencia característica (fc). Todas estas variables son de tipo continuo.

\section{RESULTADOS}

En total, se incluyeron 18 pacientes con cáncer invasivo de cérvix. Adicionalmente, en los espectros de la EIE se observó la repetición de una curva de resistividad en función de la frecuencia con un patrón característico, fácilmente reconocible y diferente a los patrones observados en estudios anteriores en epitelios escamosos y columnares sanos (figuras 1 y 2). Además, a bajas frecuencias (menores de $10 \mathrm{kHz}$ ) la resistividad del líquido extracelular de los tejidos con carcinoma invasivo presentó un valor $(\mathrm{R}=4,35+/-2,26[\Omega-\mathrm{m}])$ y la resistividad del líquido intracelular fue $(\mathrm{S}=2,44+/-1,11[\Omega-\mathrm{m}])$. El parámetro fc, frecuencia a la cual la corriente es capaz de penetrar la membrana celular y pasar a través de los espacios intra y extracelular, dio un valor de 199,47+/-128,73 (kHz) (tabla 1). 
Figura 1. Curvas de resistividad eléctrica de tejidos cervicales afectados por carcinoma invasivo.

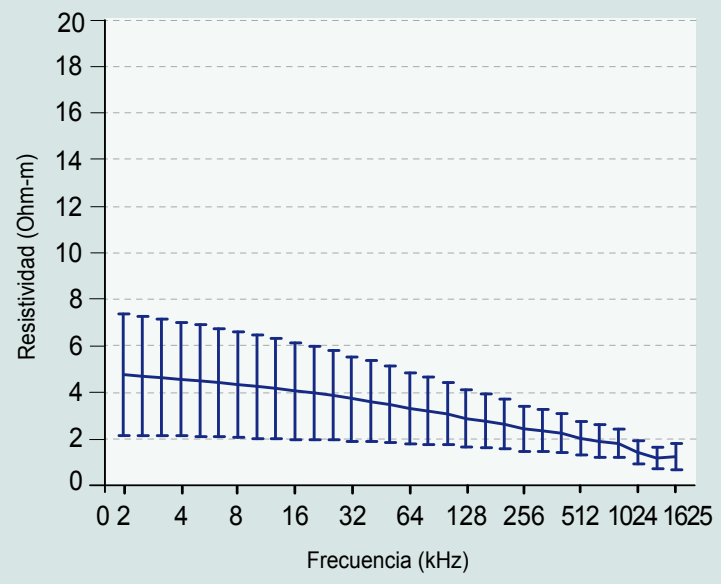

Figura 2. Curvas de resistividad de tejidos cervicales: escamoso normal $(\mathbf{\Delta})$, carcinoma invasivo $(\mathbf{x})$, columnar normal (•).

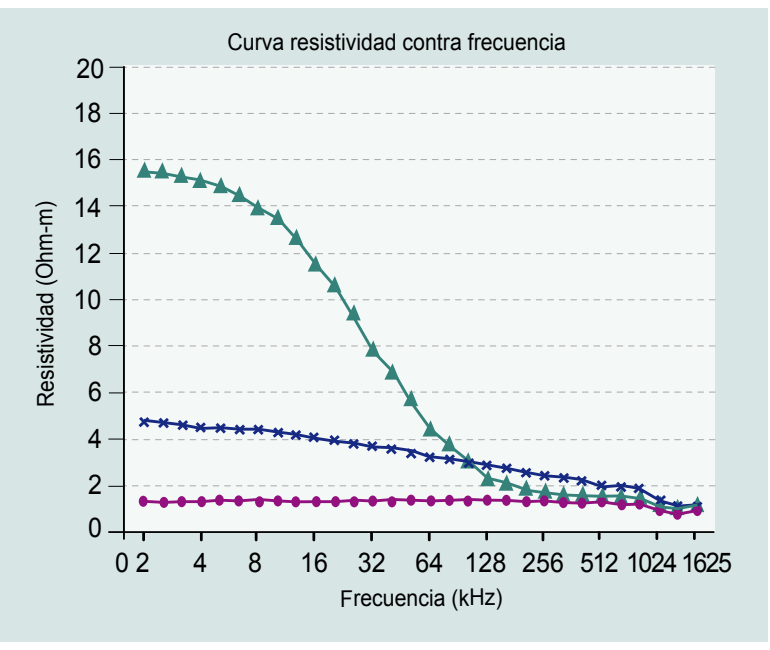

\section{DISCUSIÓN}

Diferentes estudios encaminados a detectar neoplasias intraepiteliales en tejidos cervicales, aplicando la técnica de la EIE, han demostrado que los espectros de impedancia cambian cuando estos tejidos se encuentran en fases precancerosas. ${ }^{6,10}$

De acuerdo con lo anterior, las curvas correspondientes a los espectros de impedancia para carcinomas invasivos del cuello uterino, halladas en esta investigación, se diferencian de manera fácil de las curvas obtenidas en estudios previos de tejidos escamosos y columnares sanos, así como de tejidos con lesiones neoplásicas tipo NIC 1 y NIC 2-3.,6,10,11 A propósito de lo anterior, las curvas de resistividad en tejidos con carcinomas invasivos presentan una forma característica que se reconoce porque tiene valores que son entre 4 y 5 veces menores que la resistividad del líquido extracelular de los tejidos escamosos sanos $(\mathrm{R}=24,31+/-11,26[\Omega-\mathrm{m}])$. Esta diferencia va disminuyendo con el aumento progresivo de la frecuencia, lo cual se ve reflejado porque a frecuencias superiores a los $100 \mathrm{kHz}$ la resistividad de ambos tejidos continúa descendiendo, no obstante lo hace más rápidamente la resistividad del escamoso sano que la de los tejidos con carcinoma invasivo. Se observa también que por encima de esta frecuencia, cuando parte de la corriente circula por el citoplasma, la resistividad del líquido intracelular del tejido con carcinoma invasivo de cérvix $(\mathrm{S}=2,44+/-1,11[\Omega-\mathrm{m}])$ es mayor que la del escamoso sano ( $\mathrm{S}=2,17+/-1,68[\Omega-\mathrm{m}])$ por un factor que varía entre 1,1 y 1,5 (tabla 1 ).

Igualmente, en todo el rango de frecuencias entre $2 \mathrm{kHz}$ y 1,62 MHz, la resistividad de los tejidos afectados por carcinoma invasivo es mayor que la de los tejidos columnares normales. Para frecuencias menores a los $10 \mathrm{kHz}$ la resistividad del carcinoma invasivo es mayor que la del tejido columnar sano por un factor entre 2 y 3 , aunque su diferencia va disminuyendo con el aumento de la frecuencia (figura 2). La frecuencia característica es mayor en tejidos con cáncer invasivo $(199,47 \mathrm{kHz})$ que en tejidos escamosos sanos $(6,25 \mathrm{kHz})$, pero menor que en tejidos columnares sanos (301,03 kHz).

Además de los datos expuestos, los espectros de impedancia que se han obtenido en este estudio concuerdan con los resultados reportados en otras investigaciones. ${ }^{4-7}$ Aunque en la literatura son escasos los estudios para tejidos con carcinoma invasivo, los resultados de esta investigación permiten observar la tendencia de dichos tejidos a disminuir la resistividad extracelular, a aumentar la resistividad intracelular y a presentar alteraciones que pueden ser explicadas por los cambios bioquímicos y estructurales de las células cancerosas.

Una característica común en todos los estudios es el reporte de la disminución progresiva en la resistividad 


\begin{tabular}{|l|l|c|c|c|c|c|}
\hline \multicolumn{5}{|c|}{ Tabla 1. Medidas promedio y desviación estándar de los parámetros de espectroscopia de impedancia } \\
en tejidos cervicales y cáncer invasivo. \\
\hline Parámetros & $\begin{array}{c}\text { Escamoso } \\
\text { normal }\end{array}$ & DS & $\begin{array}{c}\text { Tejidos } \\
\text { Columnar } \\
\text { normal }\end{array}$ & DS & $\begin{array}{c}\text { Cáncer } \\
\text { invasivo }\end{array}$ & DS \\
\hline $\mathrm{R}_{0}(\mathrm{Ohm}-\mathrm{m})$ & 24,31 & 11,26 & 1,71 & 0,59 & 4,35 & 2,26 \\
\hline $\mathrm{R}_{\infty}(\mathrm{Ohm}-\mathrm{m})$ & 1,99 & 1,46 & 1,21 & 0,28 & 1,41 & 0,57 \\
\hline $\mathrm{R}(\mathrm{Ohm}-\mathrm{m})$ & 24,31 & 11,26 & 1,71 & 0,59 & 4,35 & 2,26 \\
\hline $\mathrm{S}(\mathrm{Ohm}-\mathrm{m})$ & 2,17 & 1,68 & 2,99 & 0,97 & 2,44 & 1,11 \\
\hline fc $(\mathrm{kHz})$ & 6,25 & 3,62 & 301,03 & 162,53 & 199,47 & 128,73 \\
\hline
\end{tabular}

"Tomado de Olarte, et al. 2007.

de la matriz extracelular del tejido cervical cuando se presentan lesiones neoplásicas que van desde NIC 1 hasta NIC 2-3 y cáncer invasivo. Según Brown y colaboradores, la disminución progresiva de la resistividad en tejidos lesionados está asociada con la pérdida de las capas de células aplanadas cercanas a la superficie del epitelio escamoso, las alteraciones en la arquitectura, la organización del tejido, la pérdida de cohesión celular debida a la ruptura de los puentes intercelulares y con el aumento del espacio intersticial. ${ }^{4-7,11,12}$

También se presenta un incremento en la resistividad del líquido intracelular asociado con el aumento en el grado de la lesión. Esto se puede explicar por las alteraciones nucleares, por la presencia de núcleos pleomórficos de gran tamaño que alteran la relación núcleo/citoplasma y por el aumento en la densidad de la cromatina. Todos estos factores contribuyen a estrechar las rutas de la corriente a nivel intracelular que, a pesar del aumento sostenido en la concentración de sodio en presencia de cáncer invasivo, hacen que dicha resistividad sea ligeramente mayor que en tejidos escamosos sanos.

Ante la inexistencia de información específica sobre resistividad de tejidos con carcinoma invasivo, se ha tomado como referencia para la comparación de resultados los datos reportados por Brown para NIC 2-3. Se observó que no se presenta una diferencia estadísticamente significativa entre los valores de la resistividad del líquido extracelular $(\mathrm{R}=4,35+/-2,26[\Omega-\mathrm{m}])$ en carcinoma invasivo y los valores $(\mathrm{R}=3,85+/-2,89[\Omega-\mathrm{m}])$ en NIC
2-3 $\left(P_{\text {-value }}=0,9\right)$. Sin embargo, sí se presenta una diferencia estadísticamente significativa entre la resistividad del líquido intracelular ( $\mathrm{S}=2,44+/-1,11$ $[\Omega-\mathrm{m}])$ del cáncer invasivo y la reportada para NIC 2-3 $\left(\mathrm{S}=6,1+/-2,57[\Omega\right.$-m] $)(P$-value $<0,01) .{ }^{5}$ Esto permite plantear que las alteraciones estructurales en la matriz extracelular en una lesión tipo NIC 2-3 no se diferencian por la EIE de las que se presentan en un carcinoma invasivo pero los cambios a nivel intracelular sí pueden ser diferenciados utilizando la técnica de la EIE.

El hecho de que la resistividad extracelular de los tejidos con carcinoma invasivo $(\mathrm{R}=4,35+/-2,26$ $[\Omega-\mathrm{m}])$ sea mayor que la del tejido columnar sano $(\mathrm{R}=1,71+/-0,59[\Omega-\mathrm{m}])$ se puede explicar teniendo en cuenta que la estructura del tejido columnar no es estratificada (está constituido por una sola capa) y, por lo tanto presenta una ruta de baja resistividad para el flujo de corriente que es aún menor que la del tejido con cáncer invasivo. Otro signo indicativo útil para el estudio de los tejidos cervicales sanos y lesionados es el aumento en la frecuencia característica que se relaciona directamente con el incremento en el grado de la lesión. De acuerdo con Brown y Abdul, a medida que la lesión progresa, el parámetro fc aumenta. Se ha observado, en los trabajos realizados por el Grupo de Cáncer de Cuello Uterino y Cáncer de Mama de la Universidad de Caldas, que para epitelios escamosos sanos la frecuencia característica es de $6,25 \mathrm{kHz}^{10}$ y para los que presentan cáncer invasivo aumenta hasta 199,47 kHz mientras que en los tejidos columnares 
sanos esta frecuencia alcanza el valor de $301,03 \mathrm{kHz}$ El aumento de la frecuencia característica del epitelio escamoso cervical permite explicar el comienzo y la evolución de las lesiones de grados menores (virus del papiloma humano [VPH], NIC 1) a grados mayores (NIC 2-3). Este hecho fue reportado por Abdul y colaboradores, quienes lo infirieron a partir de la formulación de modelos matemáticos y utilizando la técnica de elementos finitos. ${ }^{4}$

\section{CONCLUSIÓN}

La EIE muestra un patrón diferente en tejidos provenientes de carcinoma invasivo del cérvix con relación al patrón del tejido cervical sano en los parámetros $\mathrm{R}$ y fc. Asimismo, los parámetros obtenidos por EIE para el líquido extracelular R no se diferencian de los obtenidos en pacientes con lesiones escamosas intraepiteliales NIC 2-3 del cérvix; sin embargo, el parámetro $S$ de la resistividad intracelular sí presenta diferencia estadísticamente significativa. Por consiguiente, se deben realizar estudios a mayor profundidad de la resistividad del líquido intracelular en busca de precisar dicha diferenciación.

\section{AGRADECIMIENTOS}

El Grupo de Investigación en Cáncer del Cuello Uterino y Cáncer de Mama de la Universidad de Caldas agradece a la Vicerrectoría de Investigaciones y Posgrados de la Universidad de Caldas por el apoyo económico y logístico brindado para la ejecución de este proyecto de investigación.

\section{REFERENCIAS}

1. Arrossi S, Sankaranarayanan R, Parkin DM. Incidence and mortality of cervical cancer in Latin America. Salud Pública Mex 2003;45:S306-14.

2. Piñeros M, Ferlay J, Murillo R. Cancer incidence estimates at the national and district levels in Colombia. Salud Pública Mex 2006;48:455-65.

3. Abdul S, Brown BH, Milnes P, Tidy JA. A clinical study of the use of impedance spectroscopy in the detection of cervical intraepithelial neoplasia (CIN). Gynecologic Oncology 2005;99:S64-6.

4. Abdul S, Brown BH, Milnes P, Tidy JA. The use of impedance spectroscopy in the detection of cervical intraepithelial neoplasia. Int J Gynecol Cancer 2006;16:1823-32.

5. Brown B. Measurement of the electrical properties of tissue - New developments in impedance and spectroscopy. IEICE Trans Inf \& Syst 2002;E85-D:2-4.

6. Brown BH, Tidy JA, Boston K, Blackett AD, Smallwood RH, Sharp F. Relation between tissue structure and imposed electrical current flow in cervical neoplasia. Lancet 2000;355:892-5.

7. Walker DC, Brown BH, Smallwood RH, Hose DR, Jones DM. Modelled current distribution in cervical squamous tissue. Physiol Meas 2002;23:159-68.

8. Walker DC, Brown BH, Hose DR, Smallwood RH. Modelling the electrical impedivity of normal and premalignant cervical tissue. Electronics Letters 2000;36:1603-4.

9. Jones DM, Smallwood RH, Hose DR, Brown $\mathrm{BH}$, Walker DC. Modelling of epithelial tissue impedance measured using three different designs of probe. Physiol Meas 2003;24:605-23.

10. Olarte-Echeverri G, Aristizábal-Botero W, Gallego-Sánchez PA, Rojas-Díaz J, Botero BE, Osorio GF. Detección precoz de lesiones intraepiteliales del cuello uterino en mujeres de Caldas (Colombia) mediante la técnica de espectroscopia de impedancia eléctrica. Rev Colomb Obstet Ginecol 2007;58:13-20.

11. Walker DC, Brown BH, Blackett AD, Tidy J, Smallwood RH. A study of the morphological parameters of cervical squamous epithelium. Physiol Meas 2003;24:121-35.

12. Barrow AJ, Wu SM. Impedance measurements for cervical cancer diagnosis. Gynecol Oncol 2007;107:S40-3. 